\title{
A Bilevel Equalizer to Boost the Capacity of Second Life Li lon Batteries.
}

\author{
Dr. Ngalula S. Mubenga \\ University of Toledo, USA
}

There is a strong interest in second life applications for the growing number of used electric vehicle (EV) batteries, but Capacity variations amongst these used cells present a problem. Even when these cells are matched for capacity, some imbalance is bound to remain, and a few lower capacity cells are also likely to develop after the pack begins its second life. Conventional cell voltage equalizers (EQU) do not address this problem, and they only provide a battery discharge capacity that is exactly equal to that of the weakest cell in the pack. This can easily result in a capacity loss of perhaps $20 \%$ to $25 \%$, or more. This indicates the need for a new class of EQUs that can provide a discharge capacity that is close to the average of the cells, instead of the weakest cell. It is proposed to call these "capacity EQUs", and the properties they must have are described. One such EQU is the bilevel equalizer (BEQ), described previously. This keynote provides an enhanced analysis of the BEQ and improved modelling methods. 